\title{
Epac2 contributes to PACAP-induced astrocytic differentiation through calcium ion influx in neural precursor cells
}

\author{
Hyunhyo Seo \& Kyungmin Lee ${ }^{*}$ \\ Department of Anatomy, Brain Science \& Engineering Institute, Kyungpook National University Graduate School of Medicine, Daegu \\ 41944, Korea
}

\begin{abstract}
Astrocytes play a critical role in normal brain functions and maintaining the brain microenvironment, and defects in astrocytogenesis during neurodevelopment could give rise to severe mental illness and psychiatric disorders. During neuro-embryogenesis, astrocytogenesis involves astrocytic differentiation of neural precursor cells (NPCs) induced by signals from ciliary neurotrophic factor (CNTF) or pituitary adenylate cyclase-activating peptide (PACAP). However, in contrast to the CNTF signaling pathway, the exact mechanism underlying astrocytic differentiation induced by PACAP is unknown. In the present study, we aimed to verify a signaling pathway specific to PACAP-induced astrocytogenesis, using exchange protein directly activated by cAMP2 (Epac2)-knockout mice. We found that PACAP could trigger astrocytic differentiation of NPCs via Epac2 activation and an increase in the intracellular calcium concentration via a calcium ion influx. Taken together, we concluded that astrocytogenesis stimulated by PACAP occurs through a novel signaling pathway independent from CNTF-JAK/STAT signaling, that is the well-known pathway of astrocytogenesis. [BMB Reports 2016; 49(2): 128-133]
\end{abstract}

\section{INTRODUCTION}

Astrocytes, glial cells characterized by glial fibrillary acidic protein (GFAP) expression, play a critical role in maintaining a microenvironment that supports neurons and also participate in the formation of the blood-brain barrier (1). Additionally, astrocytes involves in various neural and cognitive functions. During neurodevelopment, gp130-JAK/STAT3 pathways are known as the main pathways involved in astrocytogenesis, during which neu-

${ }^{*}$ Corresponding author. Tel: +82-53-420-4803; Fax: +82-53-4271468; E-mail: irislkm@knu.ac.kr

http://dx.doi.org/10.5483/BMBRep.2016.49.2.202

Received 7 October 2015, Revised 2 November 2015, Accepted 9 December 2015

Keywords: Astrocytogenesis, Calcium ion, Exchange protein directly activated by cAMP2 (Epac2), Neural precursor cells (NPCs), Pituitary adenylate cyclase-activating peptide (PACAP) ral precursor cells (NPCs) differentiate into astrocytes during the prenatal stage (2). Several neurotrophic cytokines, such as ciliary neurotrophic factor (CNTF) and leukemia inhibitory factor (LIF), function as gliogenic signals by which NPCs generate astrocytes via the JAK/STAT pathways (3).

Moreover, recent studies have established that PACAP, a ligand of the G protein-coupled PAC1 receptor, is able to trigger the differentiation of astrocytes from NPCs via cAMP-dependent signaling pathways (4). However, new insights suggest that PACAP-induced cAMP activates Epac, an exchange protein that is directly activated by cAMP, which acts as a guanine nucleotide exchange factor to activate Rap small GTPase (5). Epac1 is expressed throughout the body, while Epac2 is expressed mainly in the brain, pituitary gland, adrenal gland, and pancreas $(6,7)$ and a deficit in Epac2 causes brain dysfunction, such as impairments in memory and social interaction $(8,9)$. Additionally, studies using a global Epac agonist demonstrated that Epac regulates astrocyte differentiation via the transcription factor DREAM during neurodevelopment (10).

However, the roles of Epac2 during development remain unknown and whether Epac2 is involved in astrocytogenesis during embryogenesis, as well as the related underlying mechanism, is still unclear. Here, we investigated whether Epac2 regulates astrocyte differentiation in the brain during development, and demonstrate the pathway involved in this process, by using Epac2 global knockout (KO) mice.

\section{RESULTS}

Epac2 regulates astrocytogenesis during brain development We examined the expression of NeuN and GFAP as markers of neurons and astrocytes, respectively, in wild-type (WT) and Epac2-KO neonatal mouse brains. At postnatal day 0, cerebral GFAP expression in $\mathrm{KO}$ mice was significantly decreased, by approximately $70 \%$ as compared to WT mice (Fig. $1 \mathrm{~A}$ and $1 \mathrm{~B},{ }^{*} \mathrm{P}$ $=0.000012$ ), but not NeuN expression (Supplementary Fig. $1 \mathrm{~A})$, indicating that Epac2-deficiency causes defective astrocytogenesis without changing neuronal expression during brain development.

We then inspected two major types of astrocytes, protoplasmic and fibrous astrocytes, in the brains of WT and Epac2-KO mice using immunohistochemistry at postnatal day 0. 
Epac2-KO neonatal mice showed less intense GFAP immunoreactivity in both protoplasmic (gray matter) (Fig. 1D and 1G, *P $=0.03$ ) and fibrous (white matter) astrocytes (Fig. 1F and 1G, $* P$ $=0.04$ ) in the cornu ammonis (CA) of the hippocampus and the major forceps of the corpus callosum, respectively, than WT mice (Fig. 1C, hippocampus; Fig. 1E, major forceps). However, hippocampal NeuN expression did not differ between the two genotypes (Supplementary Fig. 1B). These data implied that Epac2 gene deficiency in the developing brain causes overall astrocytogenesis defects, rather than neurogenesis defects.

\section{Epac2 is involved in PACAP-induced astrocytogenesis}

Next, we investigated whether a lack of Epac2 affects astrocyte differentiation from neural precursor cells (NPCs) in vitro.
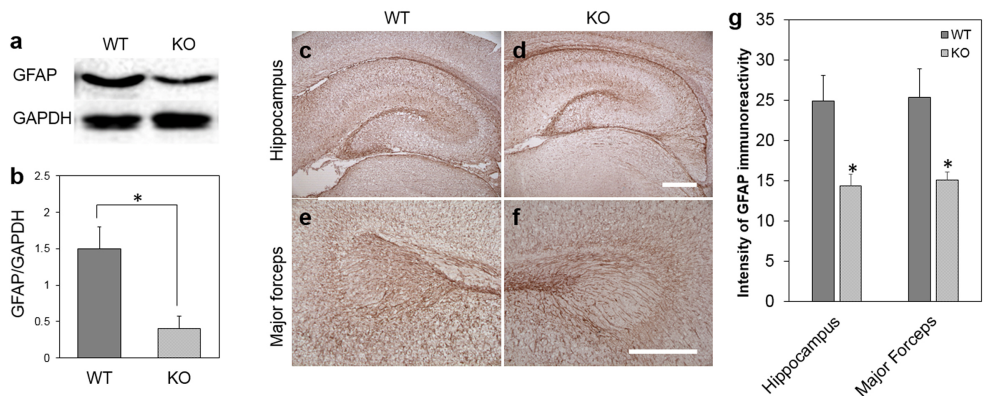

Fig. 1. GFAP expression is reduced in the neonatal brain of Epac2-knockout $(K O)$ mice. $(A, B)$ Western blot representing GFAP expression in the cerebrum of wild-type (WT) and Epac2-knockout (KO) mice at postnatal day 0. GFAP protein expression was decreased by approximately $70 \%$ in Epac2-KO neonatal brain (independent-samples Mann-Whitney $\mathrm{U}$ test, $* \mathrm{P}=0.000012 ; \mathrm{WT}=4, \mathrm{KO}=5$ in each trial, trial number $=4)$. (C-F) Immunohistochemistry images showing GFAP expression in the hippocampal CA field and major forceps of corpus callosum of WT ( $C$ and $\mathrm{E}$, respectively) and $\mathrm{KO}$ mice ( $\mathrm{D}$ and $\mathrm{F}$, respectively) at postnatal day 0 . The intensity of GFAP immunoreactivity in both the hippocampus and major forceps was lower in $\mathrm{KO}$ mice than in $\mathrm{WT}$ mice $\left(\mathrm{G},{ }^{*} \mathrm{P}=0.03\right.$ for hippocampus; ${ }^{*} \mathrm{P}=0.04$ for major forceps, independent-samples $t$-test; WT mice $=4$, slices $=8 ; \mathrm{KO}$ mice $=4$, slices $=8$ ). Scale bars $=200 \mu \mathrm{m}$.
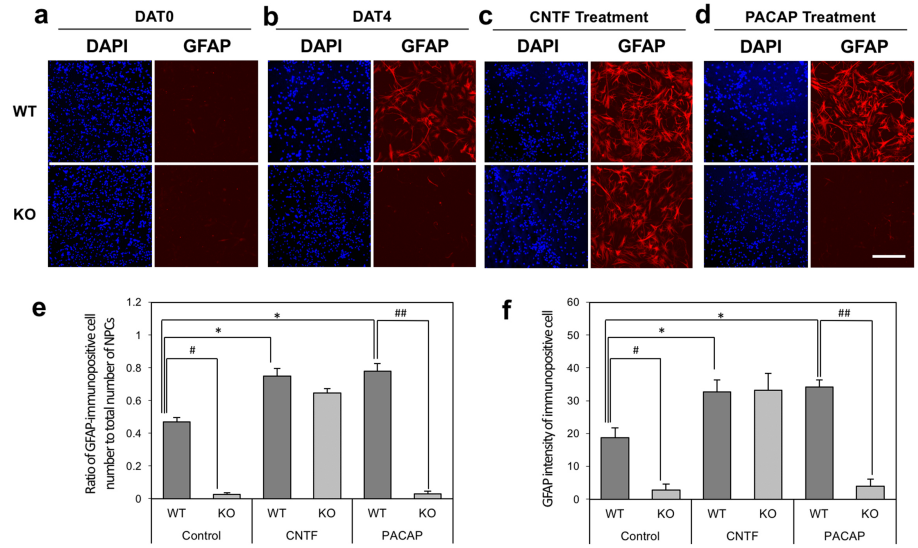

Fig. 2. Epac2 is involved in PACAP-induced astrocytogenesis. (A) GFAP immunolabeling of neural precursor cells (NPCs) at day 0 (DAT0). NPCs from neither wild-type (WT) nor Epac2-knockout (KO) mice expressed GFAP at DATO. (B) WT NPCs remarkably differentiated into astrocytes when cultured in bFGF-free media for 4 days (at DAT4), but KO NPCs showed much lower GFAP immunoreactivity than WT NPCs. (C) CNTF $(50 \mathrm{ng} / \mathrm{ml})$ induced differentiation of NPCs of both genotypes into astrocytes. (D) WT NPCs incubated in PACAP (100 nM)-treated media for 4 days expressed GFAP, but KO NPCs did not display astrocytic differentiation. (E) Ratio of GFAP-immunopositive cell number to total NPCs number. KO NPCs did not show GFAP-immunopositive astrocytes by DAT4 in bFGF-free media $\left({ }^{\#} \mathrm{P}=0.0001\right.$, independent-samples $t$-test, trial number $=8$ ). Treatment with either CNTF or PACAP increased differentiation of WT NPCs into astrocytes compared to WT NPCs without treatment at DAT4 $\left(* P=0.003\right.$ for $C N T F,{ }^{*} P=0.002$ for PACAP, independent-samples $t$-test, trial number in each experiment $\left.=8\right)$, but $K O$ NPCs did not induce astrocytic differentiation after PACAP treatment contrast to CNTF treatment ${ }^{\# \# P}=0.0002$, independent-samples $t$-test, trial number $=8$ ). (F) GFAP intensity of immunopositive cells. KO NPCs showed much lower intensity in GFAP immunoreactivity compared to WT NPCs at DAT4 in bFGF-free media ( $\mathrm{P}=0.0066$, independent-samples $t$-test). Treatment with either CNTF or PACAP increased GFAP intensity in WT NPCs compared to WT NPCs without treatment at DAT4 ( $* \mathrm{P}=0.024$ for CNTF, $* \mathrm{P}=0.007$ for PACAP, independent-samples $t$-test), but contrast to CNTF, KO NPCs did not increase GFAP immunoreactivity even after PACAP treatment compared to WT NPCs $\left({ }^{\#} \mathrm{P}=\right.$ 0.00004 , independent-samples $t$-test). Scale bar $=200 \mu \mathrm{m}$. 
Without basic fibroblast growth factor (bFGF), NPCs usually differentiate into astrocytes by day 4 in culture, in the absence of inducing factors. We compared the ability of cortical NPCs from WT and KO mice to generate GFAP-expressing astrocytes at day 0 (DAT0) in the presence of bFGF (Fig. 2A), and at day 4 (DAT4) after removing bFGF (Fig. 2B). In contrast to WT NPCs, Epac2-KO NPCs were unable to differentiate into GFAP-immunopositive astrocytes by DAT4 after removing bFGF from the culture media (Fig. 2B) as judged by the number of GFAP-immunopositive cells (Fig. $2 \mathrm{E},{ }^{\#} \mathrm{P}=0.0001$ ) and GFAP intensity (Fig. $2 \mathrm{~F},{ }^{\#} \mathrm{P}=0.0066$ ). We then investigated the Epac2-regulated upstream signaling involved in astrocytogenesis. CNTF induces astrocyte differentiation (3) and activates the MAPK and JAK/STAT pathway. Therefore, we investigated whether CNTF contributes to astrocytic differentiation of NPCs in Epac2-KO mice by adding CNTF into the culture media. Both WT and KO mice presented more GFAP-immunopositive cells (Fig. 2C and $2 \mathrm{E}, * \mathrm{P}=0.003)$ and much stronger GFAP intensity in immunopositive cells (Fig. $2 \mathrm{C}$ and $2 \mathrm{~F},{ }^{*} \mathrm{P}=0.024$ ) at DAT4 after CNTF treatment compared to those seen in non-treated WT NPCs (Fig. 2B). However, CNTF-induced astrocyte differentiation did not differ between the WT and KO NPCs (Fig. 2C, 2E, and 2F), implying that the absence of Epac2 did not affect CNTF-activated astrocytogenesis from NPCs.

Next, we examined the effect of PACAP treatment on astrocyte differentiation in NPCs. Before PACAP treatment, we assessed the expression of the PACAP receptor, PAC1R, in WT NPCs and found that all nestin-immunopositive NPCs expressed PAC1R (Supplementary Fig. 2). In the presence of PACAP, more NPCs from WT mice had differentiated into astrocytes by DAT4 (Fig. 2D), as seen by the number of GFAP- immunopositive cells (Fig. $2 \mathrm{E}, * \mathrm{P}=0.002$ ) and the GFAP intensity in the immunopositive cells (Fig. $2 \mathrm{~F},{ }^{*} \mathrm{P}=0.007$ ), than in the absence of PACAP (Fig. 2B). Contrary to CNTF, NPCs deficient in Epac2 exhibited markedly lower GFAP-immunopositive signals than did WT NPCs after treatment with PACAP (Fig. 2D) in terms of both GFAP-immunopositive cell number (Fig. $2 \mathrm{E},{ }^{\# \#} \mathrm{P}=0.0002$ ) and intensity (Fig. 2F, ${ }^{\# \#} \mathrm{P}=0.00004$ ). Thus, a genetic deficiency of Epac2 impairs PACAP-stimulated astrocytic differentiation signaling pathways, but not those stimulated by CNTF.

\section{PACAP regulates the intracellular calcium concentration of} NPCs via Epac2 during astrocytogenesis

Finally, we investigated which intermediate mediator of astrocytogenesis is involved in PACAP-Epac2 signaling pathway. In nervous tissue, calcium is involved in synaptic activity (11) and in astrocytes in particular, intracellular calcium levels play a key role in GFAP expression (10). We monitored the cytoplasmic calcium levels of NPCs loaded with the fluo-4 AM calcium indicator during astrocytic differentiation that was induced by either PACAP or CNTF. PACAP treatment caused an approximately three-fold increase in the intracellular calcium level as compared to base-line levels in WT NPCs within 1 min after treatment, but not in Epac2-KO NPCs (Fig. 3A and 3B), indicat- ing that the PACAP-induced intracellular calcium flux may be mediated by Epac2.

In contrast, calcium levels remained unchanged in NPCs of both WT and KO mice after treatment with CNTF (Fig. 3C and $3 \mathrm{D})$, suggesting that the intracellular calcium concentration of NPCs may not be involved in CNTF-induced GFAP expression. Next, we sought the source of the PACAP-induced increases in calcium levels, using WT NPCs in calcium-free culture media. Interestingly, under calcium-free conditions, PACAP treatment did not cause an increment of intracellular calcium level (Fig. 3E and 3F). Thus, PACAP-Epac2 signaling increased intracellular calcium concentration through an influx of calcium from extracellular sources, rather than from intracellularly stored calcium ion.

Next, we investigated whether the PACAP-induced intracellular calcium increase is required for astrocytogenesis. PACAP treatment barely induced astrocytogenesis under calcium-free conditions, as compared to calcium-containing conditions (Fig. 4A), indicating that the PACAP-induced increase in intracellular calcium is crucial for astrocytogenesis. We also assessed whether increased intracellular calcium levels trigger as-
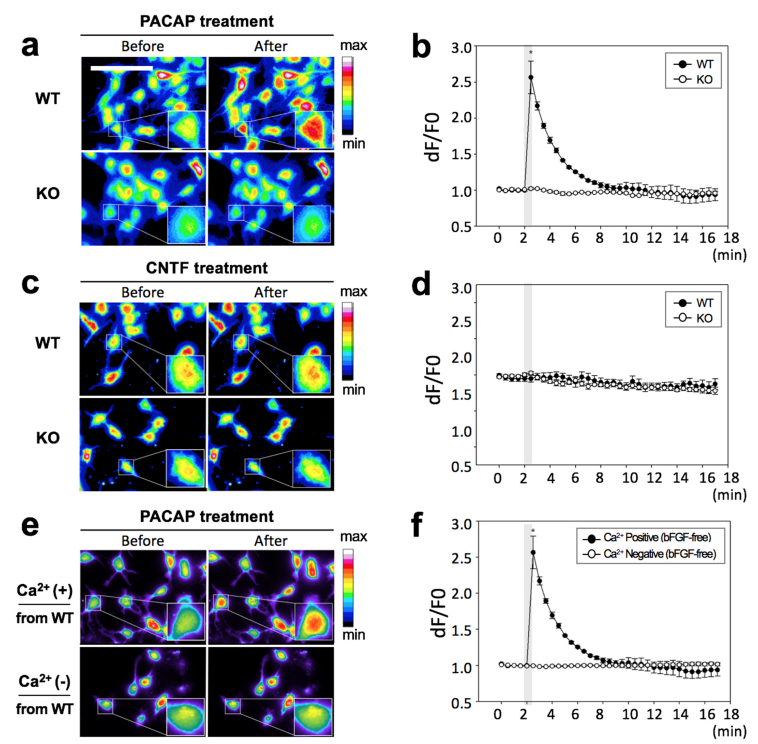

Fig. 3. PACAP regulates intracellular calcium concentration of neural precursor cells via Epac2. (A, B) Wild-type (WT) neural precursor cells (NPCs) treated with PACAP exhibited a three-fold higher concentration of calcium ions than baseline (before treatment), but intracellular calcium levels in Epac2-knockout (KO) NPCs maintained the basal level even after PACAP treatment (independent-samples Mann-Whitney U test, ${ }^{*} P=0.000054$, trial number: $W T=10$ and $\left.K O=8\right) .(C, D)$ CNTF did not influence intracellular calcium levels in NPCs of either genotypes (trial number: $\mathrm{WT}=7$ and $\mathrm{KO}=7$ ). (E, F) PACAP treatment did not induce an increase in the intracellular calcium concentration under calcium-free condition (independent-samples MannWhitney $U$ test, ${ }^{*} P=0.000019$, trial number $=15$ ), indicating that increase in calcium concentration is derived from extracellular calcium ion source. The gray bars shown in $b, d$, and $f$ indicate the duration of PACAP treatment (for $30 \mathrm{~s}$ ). Scale bar $=50 \mu \mathrm{m}$. 

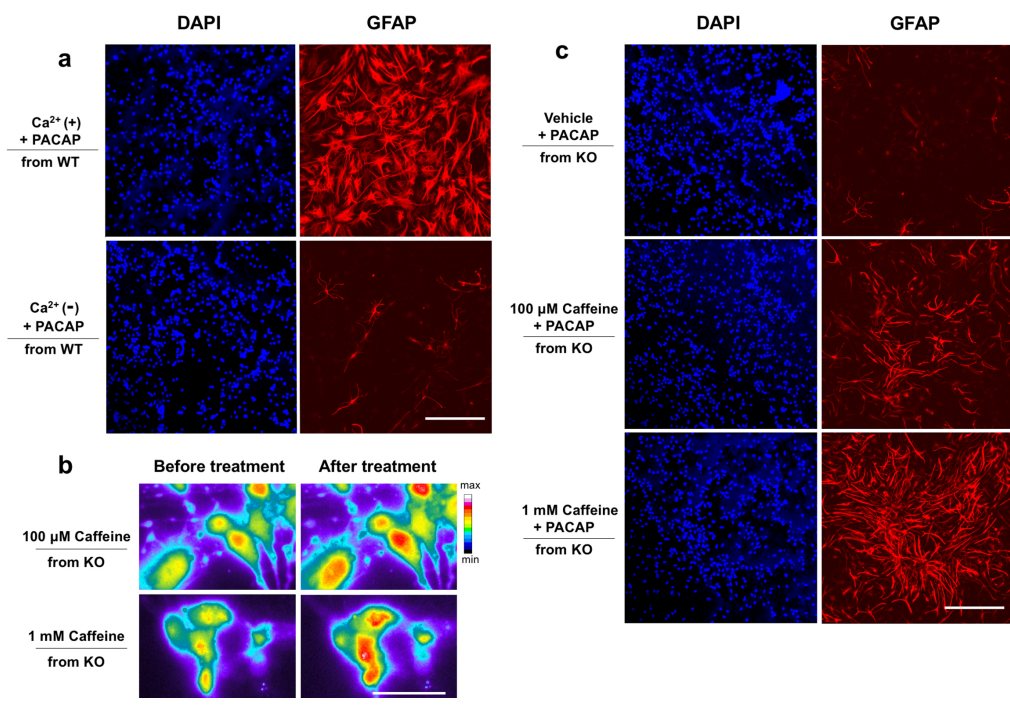

Fig. 4. Intracellular calcium increase induced by PACAP-Epac2 signaling plays a key role in astrocytogenesis. (A) Wild-type (WT) neural precursor cells (NPCs) treated with PACAP in calcium-containing media showed prominent GFAP expression, whereas WT NPCs treated with PACAP in calcium-free media exhibited markedly lower astrocytic differentiation (trial number $=3)$. (B) Application of caffeine $(100 \mu \mathrm{M}$ or 1 $\mathrm{mM}$ ) to Epac2-knockout (KO) NPCs raised the intracellular calcium concentration (trial number $=$ 3). (C) Caffeine-induced increase in intracellular calcium levels by release from intracellular calcium stores recovered PACAP-induced astrocytogenesis in Epac2-KO NPCs in a dose-dependent manner, as compared to vehicle-treated $\mathrm{KO}$ NPCs (trial number $=3$ ). Scale bars $=200 \mu \mathrm{m}$ for A and C; Scale bar $=25 \mu \mathrm{m}$ for B. trocytogenesis in Epac2-KO NPCs after PACAP treatment, by applying caffeine to Epac2-KO NPCs, as caffeine increases intracellular calcium levels by inducing calcium release from intracellular stores in mammalian neurons (12). Caffeine treatment $(100 \mu \mathrm{M}$ or $1 \mathrm{mM})$ of Epac2-KO NPCs induced an intracellular calcium concentration increase in KO NPCs within 1 min (Fig. $4 \mathrm{~B})$ and recovered astrocytogenesis in a dose-dependent manner after PACAP treatment, as compared to vehicle application (Fig. 4C).

Therefore, Epac2 is stimulated by PACAP, but not by CNTF, causing an influx of extracellular calcium into NPCs, leading to astrocytic differentiation and GFAP expression. Moreover, our data indicated that astrocytogenesis from NPCs follow two distinct signaling pathways: the PACAP-Epac2-calcium ion pathway and the CNTF signaling pathway.

\section{DISCUSSION}

We here investigated the role of Epac2 in astrocyte differentiation during neurodevelopment, although Epac2 is expressed in the cortex, hippocampus, and striatum of adult mouse brain (Supplementary Fig. 3A and 3B). We found that Epac2-deficiency impaired PACAP-induced astrocytic differentiation of NPCs without affecting neuronal differentiation, and that an increase in intracellular calcium levels is crucial in the PACAP-Epac2 signaling pathway-induced astrocytogenesis. Previous studies have demonstrated that astrocytogenesis involves the PACAP-Epac signaling pathway $(10,13)$. However, these studies could not differentiate between the role of Epac1 or Epac2, because they used a pan-Epac agonist, although each subtype of Epac has a different functional role in the brain. Our data imply a key role for Epac2 in the increase of intracellular calcium levels during GFAP expression in PACAP-induced astrocytogenesis, but not in CNTF-induced astrocytogenesis.
These findings from Epac2-KO mice imply that abnormalities in Epac2 functions could result in dysfunction of astrocytes in the formation of the blood-brain barrier, support of energy metabolism, neurotransmitter-related roles, ion concentration regulation, etc., in nervous tissues (1). This could lead to cognitive dysfunction $(14,15)$ or psychiatric disorders $(16,17)$.

Although it has been reported that PACAP, as an Epac agonist, regulates differentiation of neural stem cells into astrocytes (10), some matters require clarification. First, it is not clear whether the PACAP signaling pathway functions distinctly from the CNTF-JAK/STAT pathway as the main route for astrocyte differentiation. Second, the intermediate signaling molecule in GFAP expression stimulated by PACAP-Epac2 activation remains unidentified. CNTF modulates NPC proliferation, and regulates the astrocytic differentiation of these cells via the JAK/STAT pathway (18). Here, we found that Epac2-deficiency in NPCs did not affect CNTF-induced astrocytic differentiation of NPCs, indicating that intracellular signaling for astrocytogenesis via Epac2 is independent of the CNTF-JAK/STAT pathway.

Cytoplasmic calcium ions in NPCs play pivotal roles in cell proliferation, cell death, and differentiation $(19,20)$. Among factors inducing astrocytogenesis, PACAP has been shown to regulate intracellular calcium levels by mobilizing intracellularly stored calcium and modulating an extracellular calcium influx $(4,21)$, as was also supported by our study. Cytoplasmic calcium levels in WT NPCs increased three-fold via an influx of extracellular calcium after PACAP treatment and this increase was crucial to PACAP-induced astrocytogenesis. In contrast to WT NPCs, Epac2-KO NPCs showed no difference in cytoplasmic calcium concentration before and after PACAP treatment. However, caffeine-induced increased intracellular calcium levels rescued PACAP-induced astrocytogenesis in Epac2-KO NPCs. Taken together, these results indicate that, during astrocytic differentiation of NPCs, the PACAP-Epac2-mediated 
pathway controls calcium influx for GFAP expression. Although further studies are necessary to identify the mechanism underlying the stimulation of calcium influx via PACAP-activated Epac2, it remains possible that Epac2 may regulate whole-cell currents and surface expression of calcium channels by controlling the delivery of calcium channels to the plasma membrane or degradation of calcium channels via the proteasome or lysosome. Furthermore, in the present study we found that CNTF did not mediate intracellular calcium fluctuation during astrocytogenesis. Future studies should ascertain functional differences between the PACAP-mediated pathway and the CNTF-mediated pathway during astrocytogenesis.

This is the first report of the role of Epac2 in regulating differentiation of NPCs into astrocytes induced by PACAP, not by the CNTF-JAK/STAT pathway. These findings will facilitate the development of therapeutic agents targeting astrocytic dysfunction or malformation of gliosis that is observed in many brain disorders, such as dementia and psychiatric disorders.

\section{MATERIALS AND METHODS}

\section{Animals}

All experiments and animal protocols were carried out in accordance with the guidelines for the Care and Use of Laboratory Animals of Kyungpook National University, and pain and discomfort to animals were minimized.

The Epac2-KO mouse was generated by placing loxP sites containing a Neo-cassette around a 9.6-kb DNA fragment of exon 3 of mouse Epac2 (8), and the lack of Epac2 expression was confirmed by reverse transcriptase polymerase chain reaction (PCR) analysis using the following three primers: mCG2- I2F, 5'CGTCTTACTCTAGAAACGAC-3', mCG2-E3F, 5'-TGTTTCGCC ATGGGGATATTG-3', and mCG2-I34R, 5'-CTGGTGCTCACA CCTCGTAC-3'. The PCR products were a 300 -bp single band (KO), a 600-bp single band (WT), or both bands (heterozygote).

\section{Cell culture}

Neural stem cells were isolated from the cortexes of mice at embryonic day 17 and were then cultured in the presence of 20 $\mu \mathrm{g} / \mathrm{ml}$ bFGF (10018, PeproTech, Rocky Hill, NJ) in DMEM (Gibco, Thermo Fisher, Waltham, MA) with 1X N2 supplement (Gibco, Thermo Fisher, Waltham, MA) for 7 days. Culture media were replaced on alternate days and cells were seeded at a density of $2 \times 10^{4}$ cells $/ \mathrm{cm}^{2}$ on poly L-lysine coated $13 \varnothing$ cover glasses, and were acclimated for $24 \mathrm{~h}$ before treatment with reagents. For astrocyte differentiation, cells were cultured in DMEM with N2 or calcium-free DMEM (Gibco, Thermo Fisher, Waltham, MA) with $\mathrm{N} 2$, which was then replaced with bFGF-free DMEM with N2 and a differentiation factor, such as 100 nM PACAP (A1439, Sigma- Aldrich, St Louis, MO) or 50 ng/ml CNTF (1203158, PeproTech) for 4 days. To assay the intracellular calcium increase in Epac2-KO NPCs, $100 \mu \mathrm{M}$ or $1 \mathrm{mM}$ caffeine (C0750, Sigma- Aldrich, St Louis, MO) was applied to cells, along with 100 nM PACAP, for 4 days.

\section{Immunocytochemistry}

Cells were fixed with $4 \%$ paraformaldehyde (PFA) in phosphate-buffered saline (PBS) for $20 \mathrm{~min}$ at room temperature, and were then permeabilized with $0.1 \%$ Triton X-100 for $15 \mathrm{~min}$. Subsequently, cells were incubated in 3\% BSA in PBS for $1 \mathrm{~h}$. Pretreated cells were incubated with primary antibodies against GFAP (1 : 1,000 dilution, DAKO, Glostrup, Denmark) polyclonal antibody at $4^{\circ} \mathrm{C}$ overnight, after which they were incubated with an Alexa Fluor-conjugated secondary antibody (1:500 dilution, Molecular Probes, Eugene, OR) at room temperature for $1 \mathrm{~h}$. Fluorescence was detected under a laser confocal microscope (Zeiss LSM 5 Exciter, Zeiss, Oberkochen, Germany) (22). GFAP intensity was measured by using Image J $1.47 \mathrm{~V}$ (http://imagej.nih.gov/ij).

\section{Intracellular calcium dynamics measurement}

Calcium concentration was measured by using the calcium indicator Fluo-4 AM (F-14201, Molecular Probes). Specifically, 5 $\mu \mathrm{M}$ Flou-4 AM in phenol-red-free DMEM with Pluronic F-127 (P-3000MP, Molecular Probes) was added to the cell cultures, and cells incubated at $37^{\circ} \mathrm{C}$ for 30 min prior to experiments. In calcium-negative assays, the culture media were replaced by calcium-free DMEM containing Fluo-4 AM. Cells were mounted in the chamber on the temperature- and carbon dioxide- controlled stage of a Nikon Ti-E inverted microscope (Nikon, Tokyo, Japan). Then, a final concentration of $1 \mu \mathrm{M}$ PACAP, $0.5 \mu \mathrm{g} / \mathrm{ml}$ $\mathrm{CNTF}$, or $100 \mu \mathrm{M}$ or $1 \mathrm{mM}$ caffeine with PACAP was added to the culture, via a peristaltic pump (EYELA MP3, Tokyo, Japan), and calcium ion levels monitored by time-lapse photography. All systems were controlled by MetaMorph software (Molecular Devices, Sunnyvale, CA).

\section{Preparation of brain tissue}

The brains of neonatal mice were dissected for immunohistochemistry. To prepare paraffin sections, the brain tissue was post-fixed with $4 \%$ PFA at $4{ }^{\circ} \mathrm{C}$ for $48 \mathrm{~h}$ before dehydration with $30 \%$ sucrose. For western blotting, whole brains of neonatal littermate mice were dissected after decapitation, frozen in liquid nitrogen, and then stored at $-80^{\circ} \mathrm{C}$ until required.

\section{Immunohistochemistry}

The mouse brains were coronal-sectioned at a 3- $\mu \mathrm{m}$ thickness and tissues were rehydrated with a series of $100 \%, 90 \%, 80 \%$, $70 \%$, and $50 \%$ ethanol at room temperature for $30 \mathrm{~s}$, after deparaffinization with xylene for 10 min, twice. After PBS washing and blocking with $4 \%$ normal goat serum and re-permeabilization with $0.3 \%$ Triton $\mathrm{X}-100$ in PBS for $2 \mathrm{~h}$, tissues were incubated with anti-GFAP (1:1,000 dilution, DAKO) polyclonal antibody, at $4^{\circ} \mathrm{C}$ overnight. GFAP immunodetection was performed with the ABC kit (VECTASTAIN Elite ABC Kit, PK-6101, Vector Laboratories, Burlingame, MA) and DAB chromogen (3, 3'-diaminobenzidine HRP substrate, SK-4100, Vector Laboratories). The slides were covered with Vectamount after dehydration. GFAP intensity was measured by using Image J 1.47v (http:// 
imagej.nih.gov/ij).

\section{Western blotting}

Western blotting was performed with whole brain lysate homogenized in RIPA buffer including EDTA-free protease inhibitors (Roche, Basel, Switzerland). Thirty micrograms of total protein was loaded into each well of a sodium dodecyl sulfate-polyacrylamide gel and proteins electrophoretically separated under $80 \mathrm{~V}$, after which the proteins were transferred to a nitrocellulose membrane. After blocking with 5\% skim-milk, the membrane was incubated at $4^{\circ} \mathrm{C}$ overnight with anti-GFAP $(1: 5,000$, clone G-A-5; Sigma) monoclonal antibody, or anti-GAPDH (1:5,000 dilutions, Santa Cruz Biotechnology, TX) polyclonal antibody. The blots were visualized using a SuperSignal West Femto Maximum Sensitivity substrate (Thermo Fisher) (23). Data were normalized to GAPDH, using Image J 1.47v (http://imagej.nih.gov/ij).

\section{Statistical analysis}

The data from western blot and intracellular calcium dynamics measurement did not fit a Gaussian distribution and we therefore used the independent-samples Mann-Whitney $U$ test for analysis. The data from immunohistochemistry and immunocytochemistry were analyzed using independent-samples $t$-tests. All of the statistical analyses were conducted with Statistical Package for Social Science v22 (SPSS, IBM, USA). Differences were considered statistically significant at $P<0.05$. Error bars indicate the standard error of the mean (S.E.M).

\section{ACKNOWLEDGEMENTS}

K. Lee was supported by the Basic Science Research Program of the National Research Foundation of Korea (NRF), funded by the Ministry of Education, Science, and Technology (grant\# NRF-2013R1A1A3010216 and grant\# NRF-2013R1A3A1072 702). H. Seo was supported by a BK21 Plus grant funded by the Ministry of Education of Korea (grant\# 21A20132212094).

\section{REFERENCES}

1. Wang DD and Bordey A (2008) The astrocyte odyssey. Prog Neurobiol 86, 342-367

2. Park JK, Williams BP, Alberta JA and Stiles CD (1999) Bipotent cortical progenitor cells process conflicting cues for neurons and glia in a hierarchical manner. J Neurosci 19, 10383-10389

3. Bonni A, Sun Y, Nadal-Vicens M et al (1997) Regulation of gliogenesis in the central nervous system by the JAK- STAT signaling pathway. Science 278, 477-483

4. Vaudry D, Falluel-Morel A, Bourgault S et al (2009) Pituitary adenylate cyclase-activating polypeptide and its receptors: 20 years after the discovery. Pharmacol Rev 61, 283-357

5. Breckler M, Berthouze M, Laurent AC, Crozatier B, Morel E and Lezoualc'h F (2011) Rap-linked cAMP signaling Epac proteins: compartmentation, functioning and disease implications. Cell Signal 23, 1257-1266
6. Gloerich M and Bos JL (2010) Epac: defining a new mechanism for cAMP action. Annu Rev Pharmacol Toxicol 50, 355-375

7. Grandoch M, Roscioni SS and Schmidt M (2010) The role of Epac proteins, novel cAMP mediators, in the regulation of immune, lung and neuronal function. Br J Pharmacol 159, 265-284

8. Lee K, Kobayashi $\mathrm{Y}$, Seo $\mathrm{H}$ et al (2015) Involvement of cAMP-guanine nucleotide exchange factor II in hippocampal long-term depression and behavioral flexibility. Mol Brain 8, 38

9. Fernandes HB, Riordan S, Nomura T et al (2015) Epac2 Mediates cAMP-Dependent Potentiation of Neurotransmission in the Hippocampus. J Neurosci 35, 6544-6553

10. Cebolla B, Fernandez-Perez A, Perea G, Araque A and Vallejo M (2008) DREAM mediates cAMP-dependent, $\mathrm{Ca}^{2+}$-induced stimulation of GFAP gene expression and regulates cortical astrogliogenesis. J Neurosci 28, 6703- 6713

11. Brini M, Cali T, Ottolini D and Carafoli E (2014) Neuronal calcium signaling: function and dysfunction. Cell Mol Life Sci 71, 2787-2814

12. Tsai TD and Barish ME (1995) Imaging of caffeine-inducible release of intracellular calcium in cultured embryonic mouse telencephalic neurons. J Neurobiol 27, 252- 265

13. Vallejo M (2009) PACAP signaling to DREAM: a cAMP- dependent pathway that regulates cortical astrogliogenesis. Mol Neurobiol 39, 90-100

14. Srivastava DP, Jones KA, Woolfrey KM et al (2012) Social, communication, and cortical structural impairments in Epac2-deficient mice. J Neurosci 32, 11864-11878

15. Yang Y, Shu X, Liu D et al (2012) EPAC null mutation impairs learning and social interactions via aberrant regulation of miR-124 and Zif268 translation. Neuron 73, 774-788

16. Sandau US, Alderman Z, Corfas G, Ojeda SR and Raber J (2012) Astrocyte-specific disruption of SynCAM1 signaling results in ADHD-like behavioral manifestations. PLoS One 7, e36424

17. Morgan JT, Barger N, Amaral DG and Schumann CM (2014) Stereological study of amygdala glial populations in adolescents and adults with autism spectrum disorder. PLoS One 9, e110356

18. Richardson PM (1994) Ciliary neurotrophic factor: a review. Pharmacol Ther 63, 187-198

19. Teh DB, Ishizuka T and Yawo H (2014) Regulation of later neurogenic stages of adult-derived neural stem/progenitor cells by L-type $\mathrm{Ca}^{2+}$ channels. Dev Growth Differ 56, 583-594

20. Glaser T, de Oliveira SL, Cheffer A et al (2014) Modulation of mouse embryonic stem cell proliferation and neural differentiation by the P2X7 receptor. PLoS One 9, e96281

21. Payet MD, Bilodeau L, Breault L et al (2003) PAC1 receptor activation by PACAP-38 mediates $\mathrm{Ca}^{2+}$ release from a CAMP-dependent pool in human fetal adrenal gland chromaffin cells. J Biol Chem 278, 1663-1670

22. Eom SA, Kim DW, Shin MJ et al (2015) Protective effects of PEP-1-Catalase on stress-induced cellular toxicity and MPTP-induced Parkinson's disease. BMB Rep 48, 395-400

23. Lu X, Hu X, Song L et al (2015) The SH2 domain is crucial for function of Fyn in neuronal migration and cortical lamination. BMB Rep 48, 97-102 